\title{
Mitochondrial Quality Control: Impact on Aging and Life Span - A Mini-Review
}

\author{
Heinz D. Osiewacz Dominik Bernhardt \\ Faculty for Biosciences and Cluster of Excellence 'Macromolecular Complexes' Frankfurt, Institute of Molecular \\ Biosciences, Johann Wolfgang Goethe University, Frankfurt, Germany
}

\section{Key Words}

Mitochondria $\cdot$ Aging $\cdot$ Reactive oxygen species · Protein quality control · Mitophagy · Apoptosis · Mitochondrial dynamics

\begin{abstract}
A fundamental impact of mitochondria on biological aging has been suggested decades ago. One prominent theory explains aging as the result of the age-related accumulation of random molecular damage of biomolecules resulting from the reaction of reactive oxygen species, the majority of which are generated in mitochondria. Although this concept appeared to be very attractive and strongly influenced aging research, in recent years more and more data accumulated which seem to contradict this theory. However, since these data are derived from reductionist approaches and do not integrate the various components and pathways which are affected as a result of a primary experimental intervention, they are prone to misinterpretation and have to be taken with some caution. Here, after a general introduction of mitochondrial function, we discuss the relevance of various pathways which are involved in keeping mitochondria functional over time. Moreover, we provide examples which emphasize the importance of a critical interpretation of experimental data and the necessity for a holistic analysis of the
\end{abstract}

aging process. The success of such a systems biology approach is strongly dependent on the development of methods for data mining and an efficient analysis and modeling of the huge data sets that are raised.

Copyright $\odot 2013$ S. Karger AG, Basel

\section{Introduction}

Mitochondria are eukaryotic organelles best known for their essential role in energy transduction. In aerobic organisms, they generate most adenosine triphosphate (ATP) which is used to drive all cellular energy-consuming processes. Apart from this role, mitochondria are essential for iron/sulfur cluster biosynthesis, the synthesis of amino acids, lipid metabolism, copper homeostasis, the control of apoptosis and other processes.

Mitochondria are semi-autonomous elements, the biogenesis of which depends on the coordinated expression of the genome located in two cellular compartments: the nucleus and the mitochondrial matrix. Only about $1 \%$ of the approximately 1,000-1,200 mitochondrial proteins are encoded by the mitochondrial DNA (mtDNA). The vast majority of proteins are nuclear encoded, synthesized in the cytoplasm, imported into mitochondria and sorted to the different subcompartments of their function

\section{KARGER}

E-Mail karger@karger.com

www.karger.com/ger
Prof. Heinz D. Osiewacz, $\mathrm{PhD}$

Faculty for Biosciences and Cluster of Excellence 'Macromolecular Complexes' Frankfurt Institute of Molecular Biosciences, Johann Wolfgang Goethe University

Max-von-Laue-Strasse 9, DE-60438 Frankfurt (Germany)

E-Mail osiewacz@bio.uni-frankfurt.de 
where they may be assembled into macromolecular complexes (e.g. respiratory chain complexes). There is no de novo biogenesis of mitochondria, but they increase in number and mass as a result of fission of existing organelles and growth of these units by incorporation of newly synthesized molecules. Individual mitochondria can also fuse. Together, this kind of mitochondrial dynamics gives rise to constant changes in the mitochondrial morphology and is part of a hierarchical network of pathways involved in controlling the quality of a mitochondrial population. Finally, severely affected mitochondrial units can be degraded by mitophagy, a special form of selective autophagy $[1,2]$.

\section{Mutations in mtDNA}

Mitochondria contain their own genetic information. In most species, the mtDNA is a circular molecule and occurs in many copies in a single mitochondrion. In humans, 13 essential proteins of the respiratory chain and ATP synthase, a set of mitochondrial tRNAs and the small and large subunit of the mitochondrial ribosomal RNA are encoded on a 15.6-kbp mtDNA molecule. Age-related changes in mtDNA were first reported in a few fungi in the early days when molecular biology arose as a powerful discipline [3]. The subsequent analyses of mtDNA changes in mammals were at first rather disappointing, but with the development of polymerase chain reaction technology, extensive studies revealed age-related increases in mtDNA mutations, both point mutations as well as gross reorganizations like larger deletions [4-6]. Interestingly, the accumulation of such mutations occurring in a single mtDNA molecule is the result of clonal expansion by a yet unknown mechanism. Reaching a critical threshold, such mutations can give rise to adverse effects particularly on mitochondria in which impaired components (e.g. proteins of the respiratory chain) need to be replaced.

Experiments with transgenic mice revealed mechanistic aspects of mtDNA mutations and mitochondrial impairments in mammalian aging. A transgenic mouse ('mitochondrial mutator mouse') [7, 8] affected in proofreading activity, as a result of exchanging a critical amino acid in the exonuclease domain of polymerase $\gamma$, accumulated mitochondrial mutations and displayed an accelerated aging phenotype with the expression of various senescence markers including kyphosis, hearing loss, grey hair and loss of skeletal muscle mass (sarcopenia). In this mouse, mean and maximum life span is strongly decreased. No increases in reactive oxygen species (ROS) levels and in oxidation products of lipids, proteins and DNA were found in the mutator mouse. The premature aging phenotype appears to result from mitochondrial dysfunction triggering the activation of cytoplasmic caspase-3. While these data clearly show the strong potential of mtDNA mutations to cause impairments as they are observed in 'normal' aging, they do not prove that mtDNA mutations are the cause of 'normal' aging. In fact, in an analysis of mtDNA mutations in tissues of mice with normal polymerase $\gamma$, they were found to be much lower than in the mutator mouse [9].

\section{Generation and Scavenging of ROS}

It has been noticed early that mitochondrial respiration does not only lead to the generation of ATP but also to the superoxide anion, as a result of the transfer of a single electron to an oxygen molecule. This primary free radical can react with biomolecules in the direct vicinity or be converted to hydrogen peroxide via the activity of the scavenging enzyme superoxide dismutase (Sod). Hydrogen peroxide can cross membranes and diffuse to different cellular compartments. It can be converted to water by peroxiredoxin and catalase, respectively, or can give rise to the formation of the highly toxic hydroxyl radical. The generation and the damaging potential of mitochondrial derived ROS is the basis of the 'mitochondrial free radical theory of aging' (MFRTA) [10] which has been extensively investigated and refined during the last decades. This theory states that mitochondria generate ROS which lead to random damage of all kinds of molecules. While supported by many data, in recent years the theory became challenged by accumulating controversial results [11]. For instance, in the short-lived fungus Podospora anserina, no increase in carbonylation of the bulk of all mitochondrial proteins can be observed during aging [12]. In addition, overexpression of the gene coding for the mitochondrial localized PaSod3 leads to increased sensitivity against oxidative stress and a reduced life span, while deletion of PaSod3 does not affect life span [13]. These findings are in line with data obtained with Caenorhabditis elegans Sod deletion strains [14]. This kind of counterintuitive results obtained in different systems may be due to differences in the complex pathways involved in coping with the damaging potential of ROS. Moreover, arising from recent studies with genetically manipulated strains of $P$. anserina, the life span-decreasing effect of an upregulation of the mitochondrial MnSod (PaSod3) can be attributed to impairments in the mito- 
chondrial peroxiredoxin, the enzyme that converts hydrogen peroxide to water, and to the two mitochondrial matrix proteases ClpP and Lon 1 [13, 15]. Also, mitochondrial aconitase, which contains a redox sensitive iron/sulfur cluster, was demonstrated to be sensitive to carbonylation [12]. Loss of the activity of this protein was repeatedly reported $[16,17]$.

In cases where the deletion of a particular gene coding for one component of the ROS scavenging system, like the mitochondrial Sod, or of Sod isoforms of an organism may not lead to the expected results (i.e. reduction in life span), it has to be considered that other pathways, like autophagy/mitophagy or components of the proteolytic systems, are induced and can compensate for the loss of the scavenging capacity. Unfortunately, the identification of such compensatory processes is often not easy due to the lack of appropriate tools (e.g. antibodies). However, with the development and efficient use of high throughput techniques (genome-wide transcriptome analyses, mass spectroscopy), standardized protocols may become a routine, leading to a more holistic characterization of organisms in which specific interventions have been carried out.

\section{Protein Quality Control}

Mitochondria contain a number of different enzymes (fig. 1) that are involved in protein quality control $[1,18]$. There are chaperones leading to the refolding of misfolded proteins. Methionine reductase can reduce proteins with oxidized methionine, and proteases located in the different mitochondrial compartments degrade impaired proteins [19]. The role of the two matrix proteases PaLon 1 and $\mathrm{PaClpP}$ and of the mitochondrial inner membrane $i$-AAA protease PaIap has been studied in P. anserina. The overexpression of PaLon 1 was found to lead to mitochondria with improved respiration, a decrease in methylcarboxylation of mitochondrial proteins and a reduction in carbonylation of mitochondrial aconitase. No significant age-related difference in bulk carbonylation of mitochondrial proteins was detected by oxyblot analysis [12]. Importantly, the health span, the unimpaired period of time within the life of the organism, was increased. Consistently, deletion of PaLon 1 was found to lead to a life span reduction [20]. A strong effect on life span was also obtained in a strain in which PaIap was ablated by deletion of the corresponding gene [21]. However, surprisingly, the strain was characterized by an increased life span under standard growth conditions, although a com- ponent of the quality control system was absent. Significantly, the life span was decreased when the strain was grown on increased temperature unmasking a temperature-dependent role of this particular protease. This role seems to be relevant for a heterothermophilic and sessile organism under natural conditions with constantly changing temperatures. A similar temperature-dependent life span-decreasing effect of the $\mathrm{PaClpP}$ deletion, the gene coding for the second mitochondrial matrix protease, was recently demonstrated [22].

There are other proteolytic systems active to control mitochondrial integrity and quality. One recent pathway is mitochondrial associated degradation (MAD) that is part of the more general cytoplasmic ubiquitin proteasome system (UPS). This pathway, which resembles the endoplasmic reticulum-associated degradation (ERAD) system, leads to the degradation of proteins which are present at the outer mitochondrial membrane and are post-translationally modified by ubiquitination, extracted from the membrane and delivered to the $26 \mathrm{~S}$ proteasome, where they are finally degraded [23]. The proteins controlled by this pathway are not only genuine outer-membrane proteins but may originate from other mitochondrial subcompartments. In this case, they are retro-translocated from their site of function to the outer mitochondrial membrane. While MAD has been characterized as a component of the mitochondrial protein quality control system, no data about the relevance of the system for aging have been reported so far. However, MAD is demonstrated to play a role in Parkinson's disease where, as a consequence of a drop in the mitochondrial membrane potential, the serine/threonine kinase PINK translocates to the outer membrane and recruits the E3 ligase Parkin to mitochondria. Subsequently, Parkin leads to the ubiquitination of proteins including the mitofusins Mfn1 and Mfn2. In yeast, two other proteins involved in mitochondrial fission, Dnm1 and Fis1, are ubiquitinated by the two E3 ligases Mito 1 and March5, suggesting a control of mitochondrial dynamics by MAD and a link of this part of the protein quality control system with mitochondrial dynamics. There are many more ubiquitinated proteins for which the enzymes controlling their ubiquitination remain to be identified. For instance, in mitochondrial extracts from the mouse heart, $>100$ ubiquitinated proteins have been identified [24]. From these data, MAD and the proteasomal system emerge as an important pathway involved in mitochondrial quality control.

Apart from the enzymes described above, other proteolytic components are active in mitochondria, some of which are involved in the processing of specific proteins. 


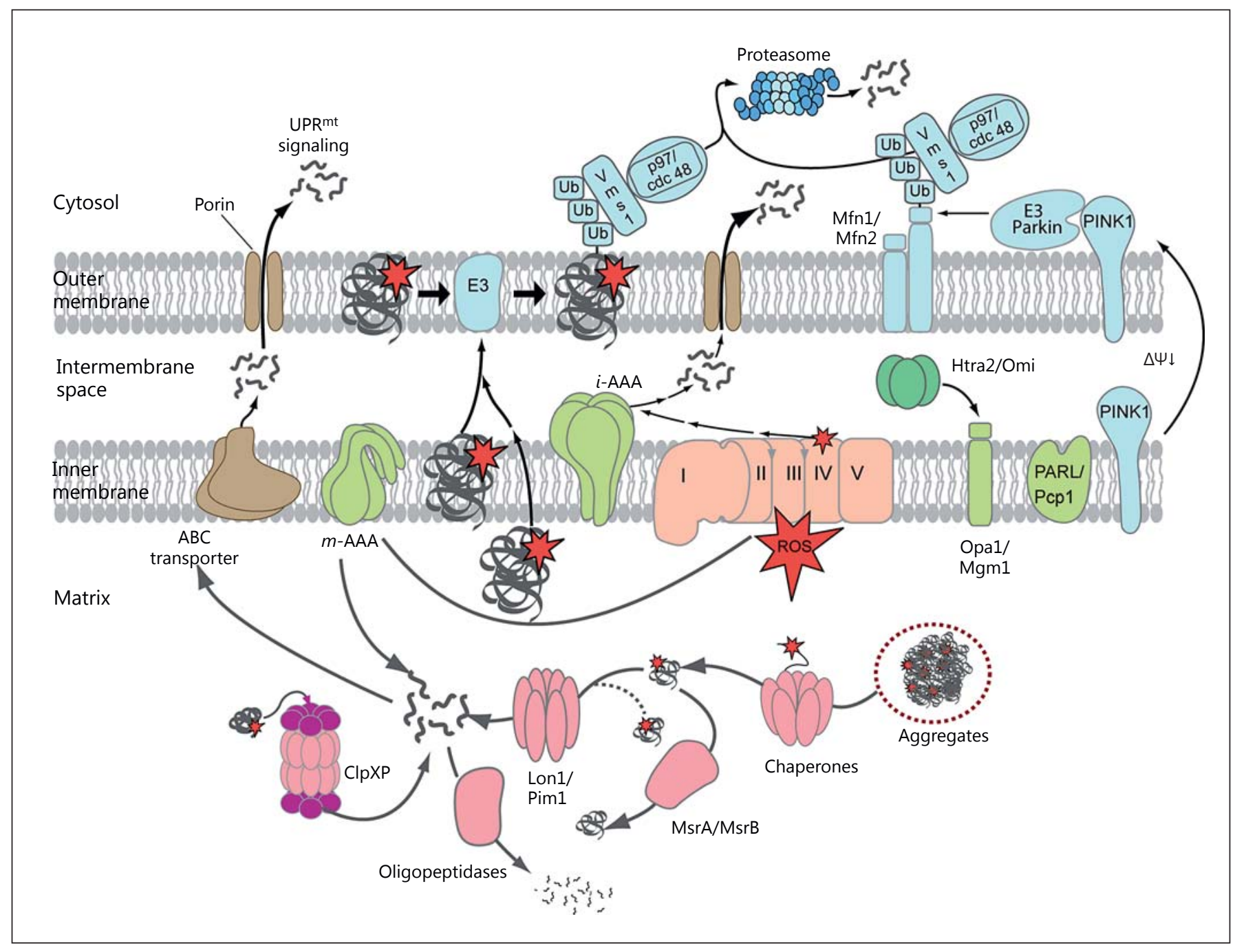

Fig. 1. Scheme depicting the complex pathways involved in mitochondrial quality control (according to [1], modified). In the mitochondrial matrix chaperones, repair enzymes (i.e. MsrA/MsrB), proteases (ClpXP, Lon1/Pim1) and peptidases are controlling the refolding, repair and degradation of damaged proteins (symbolized by a star in the protein chain). Damage may result from the reaction of the proteins with ROS generated at the respiratory chain in the inner mitochondrial membrane. Proteases in the inner membrane ( $m$-AAA, $i$-AAA, PARL) and the intermembrane space
(Htra2/Omi) control degradation and processing of proteins in the inner membrane. PINK1 (a kinase), which translocates from the inner to the outer membrane as a result of a drop of the membrane potential, E3 ligases in the outer membrane and protein complexes in the cytoplasm are involved in MAD of proteins in the outer mitochondrial membrane by the proteasome. These proteins may reach the outer membrane after retro-translocation from different mitochondrial subcompartments.
One important challenge now is the elucidation of the substrates of the different proteases. This knowledge will certainly lead to the discovery of new pathways which are of particular relevance in development, aging and agerelated diseases. Moreover, currently, detailed information about overlapping functions of the different components of the protein quality control pathways and about their redundancy and interactions is missing.

\section{Remodeling of Dysfunctional Components}

Degradation of impaired mitochondrial molecules and complexes must be followed by remodeling of the corresponding functional units. Since most mitochondrial proteins are encoded by the nuclear DNA (nDNA), the integrity of this information is strongly dependent on efficient repair systems. Nuclear encoded proteins have to be syn- 
thesized at cytoplasmic ribosomes and transported into the appropriate subcompartments of the mitochondrion where they are active. This requires the controlled transport through membranes and specific sorting processes. For some complex structures like the respiratory chain and the ATP synthase, the situation is complicated because they are encoded by the nDNA and mtDNA. Thus, mechanisms (i.e. DNA repair) keeping the integrity of DNA are important. Moreover, a coordination of providing mtDNA- and nDNA-encoded proteins is essential. While the requirements and mechanisms of the biogenesis of mitochondria as semi-autonomous organelles are studied in general in great detail, the impact of malfunctions of the individual pathways in respect to aging is basically unexplored. There is, however, no doubt that these pathways are prone to impairments and are all contributing to aging.

\section{Mitochondrial Dynamics}

The pathways discussed so far which are involved in the control of mitochondrial function are active at the molecular level. If these systems reach their limits and mitochondrial impairment increases and passes certain thresholds, a next line of quality control occurs at the organellar level via fission and fusion of mitochondria. First data about such a role were obtained with $P$. anserina and the yeast Saccharomyces cerevisiae. It was found that mitochondria of juvenile cultures are filamentous, while those of senescent cultures are punctate [25]. Furthermore, in senescent individuals of $P$. anserina, transcripts of the Dnm1 gene coding for a crucial GTPase active in mitochondrial fission were increased. Deletion of the Dnml gene in P. anserina and, consistently, in yeast were found to lead to an increased health span of the corresponding strains. Controversial results were obtained with Caenorhabditis elegans and mice. The life span of C. elegans strains, treated with Drp1 (homolog of yeast Dnm1) RNAi or harboring a Drp1 mutation, was unchanged compared to the wild type [26]. In mice, the deletion of parts of Drp1 is embryonic lethal [27]. The analyses of embryos of homozygous Drp1 deletion strains revealed different abnormalities, particularly in the forebrain. Developing embryos were found to die between embryonic day 10.5 and 12.5. Significantly, cell cultures derived from homozygous Drp1 deletion strains were healthy, demonstrating that Drp1 is dispensable for viability and proliferation of cultured cells. Embryos from Drp1-ablated mice were found to be smaller in body size, have a poorly developed liver, a thinner neural tube cell

Mitochondrial Quality Control layer and a less developed cardiac system indicating that Drp1 is essential during embryonic development. Detailed studies of these mice and of cell cultures derived from different cell types of the Drp1 deletion embryos revealed evidence of a specific importance of $\operatorname{Drp} 1$ in the nervous system. This was further validated in mice in which Drp1 deletion was restricted to the neural system by driving the expression of Cre recombinase, which leads to the deletion of part of the Drp1 gene, under the control of the neural system-specific nestin promoter. Neural cell-specific (NS) Drp1-/- mice died soon after birth due to brain hypoplasia with apoptosis. There was a clear increase in TdT-mediated dUTP nick end labeling (TUNEL)-positive neuronal cells, an indicator of apoptosis, in particular in the deep cortex layers. Embryonic fibroblasts and embryonic stem cells from these mice revealed that Drp1 is required for a normal rate of cytochrome release and caspase activation during apoptosis. Mitochondrial outer-membrane permeabilization (MOMP) leading to the release of the Smac/Diablo apoptogen appears to be independent of Drp1 activity. Taken together, the data of these studies disclose an essential function of the mitochondrial fission/fusion machinery for mouse development not allowing the loss of important components like Drp1. It remains to be shown how deletion of Drp1 affects other cell types and organs than the neural system, embryonic stem cells and embryonic fibroblast cells (e.g. skeletal cells and the heart muscle). Moreover, with respect to aging, it appears to be crucial to answer the question about potential changes of the fission/fusion machinery later in life of mammals and about their consequences as they have been demonstrated to occur in aging of wild-type strains of fungal aging models. Such studies can answer the question about the degree of conservation of the underlying mechanisms.

Overall, mitochondrial fusion is generally accepted to play an effective role in quality control. Mixing the content of impaired mitochondria with fully functional ones can result in dilution of damage and a functional organelle (fig. 2a). Fission of mitochondria is required in growing and developing systems for increasing the number of mitochondria (fig. 2b). This may specifically be required in dividing tissues to ensure that each cell is supplied with an appropriate population of mitochondria. On the other hand, fission is thought to be an important component for separating parts of an existing mitochondrion that are severely damaged or contain high amounts of damaged (e.g. aggregated proteins) components (fig. 2b). In this case, separation of this part is a prerequisite for degradation by mitophagy $[2,28]$. 
a

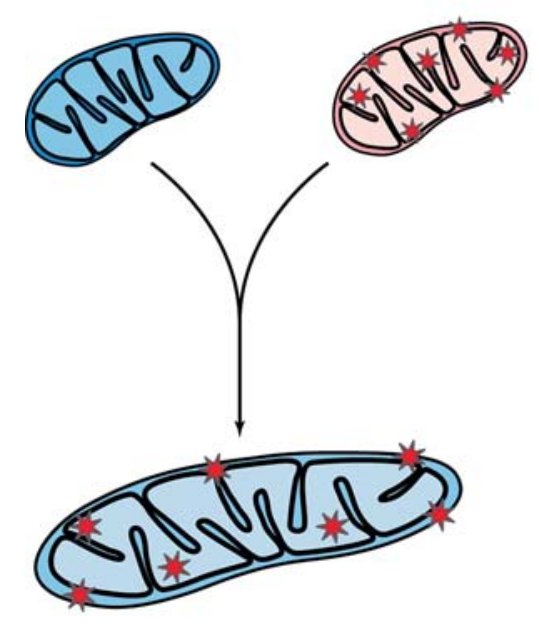

b

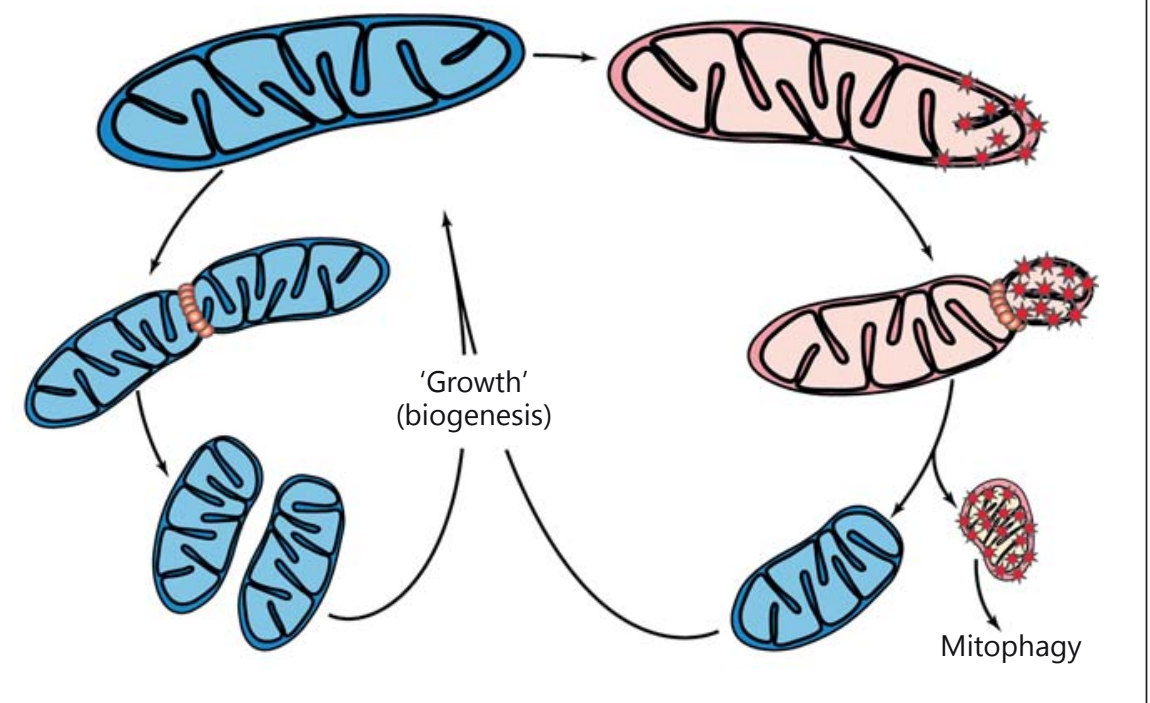

Fig. 2. The role of mitochondrial dynamics in controlling the quality of mitochondria. a Fusion of a fully functional mitochondrion (no stars) and a damaged (symbolized by stars), functionally impaired mitochondrion leads to the formation of a functional mitochondrion by content mixing and complementation of affected functions. b Mitochondrial fission (left) leading to the multiplication of mitochondria. In this example, a fully functional mitochon- drion divides into two smaller functional mitochondria which, by insertion of newly synthesized components, 'grows' to reach a larger size. On the right side, a part of an impaired mitochondrion (stars) with locally concentrated damaged components is separated. The nonfunctional, damaged unit is degraded by mitophagy. The other mitochondrion is fully functional and 'grows' by insertion of newly synthesized mitochondrial components.

\section{Mitophagy}

Mitophagy is a form of selective autophagy, in which severely impaired mitochondria are degraded $[1,2]$. This mechanism is linked to mitochondrial dynamics. Portions of a filamentous mitochondrion are separated via fission. If the resulting mitochondrion is impaired in function that is related to a decreased membrane potential, this mitochondrion is unable to fuse with another one and is engulfed by a double membrane leading to the formation of the autophagosome. Subsequently, this unit fuses with a lysosome (in animals) or the vacuole (in plants and fungi) and the content of the autophagosome including the impaired mitochondrion is degraded. While the impact of mitophagy on mitochondrial quality control and aging has been hypothesized, as yet experimental data are rather scarce. In yeast, it was shown that loss of mtDNA and the activation of the retrograde response, a pathway signaling mitochondrial impairments to the nucleus, which is involved in life span control, can lead to mitophagy. This process is believed to trigger the extended replicative life span in mtDNA-deficient strains [2].

\section{Apoptosis}

Quality control does also occur at the cellular level. In particular, in multicellular systems like mammals the role of programmed cell death (PCD), the programmed elimination of cells, is well documented to protect against cancer [29]. A role in unicellular organisms like yeast and filamentous fungi like P. anserina is less expected [30]. In these organisms, PCD is the final execution process bringing life of the individual to an end. Such a role on organismal aging is less clear in mammals. At least, data are accumulating which indicate that the induction of PCD by mitochondrial apoptosis pathways leads to cellular and organ failure and may contribute to aging and organismal death. For instance, a role of the mitochondrial transition pore (mPTP) has been identified limiting life span of the fungal aging model P. anserina [31] and mice. Cyclophilin $\mathrm{D}(\mathrm{CypD})$ increases in abundance during aging of $P$. anserina [32]. Overexpression of the gene coding for CypD leads to accelerated aging. In mice, an increase of CypD was found in gastronemius muscle in aged individuals [33]. In the 'mitochondrial mutator mouse' with 
accelerated aging, a significant loss in gastronemius and quadriceps muscle and increased apoptosis was described [34]. Mice lacking the Sirt3 deacetylase were found to express an accelerated aging phenotype that is correlated with increased mitochondrial swelling as a result of mitochondrial permeability transition pore opening. The mice were hypersensitive to heat stress and displayed cardiac hypertrophy and fibrosis [35]. In mammals, CypD thus appears to be of relevance not only for skeletal muscle function but also in the heart, connecting this pathway to one of the most important age-related causes of death in humans, cardiac failure.

The release of proapoptotic compounds from mitochondria (e.g. cytochrome c) leading to the induction of apoptosis can also result from rupture of the outer mitochondrial membrane caused by different other processes. Parkin as well as various other components like proapoptotic $\mathrm{Bcl} 2$ proteins and Drp1 interacting with the endoplasmic reticulum during endoplasmic reticulum-associated mitochondrial division (ERMD) can be involved [36]. In C. elegans, the Bcl2-like Ced9 protein which is best characterized for its function in apoptosis has recently been demonstrated to be part of a molecular switch governing the balance between $\mathrm{Ced} 9 / \mathrm{Bcl} 2$-mediated mitochondrial fusion and fission. The function depends on the interaction of Ced 9 with different proteins [37].

\section{Conclusions}

With the identification of an elaborate system of individual pathways involved in mitochondrial quality control, questions about their redundancy, interaction and their control arise. Up to now, various components of this system have rather been investigated individually to some detail using reductionist approaches (e.g. gene knock-out strategies). However, currently, we are far from knowing enough details about any single pathway. For instance, although investigated intensively over decades, the precise role of ROS and of ROS scavenging systems in aging and development is not clear at all and is currently discussed controversially. In particular, the impact of a spatial and temporal regulation of ROS levels for signaling purposes is poorly explored. Other largely open questions are the profiles of substrates recognized by the different mitochondrial proteases and, thus, of the molecular pathways affected by these important quality control components. Nevertheless, some evidence has emerged suggesting that the individual quality control pathways are part of a complex hierarchical system and that different components are induced once individual pathways are overwhelmed in their capacity. The elucidation of the different regulatory circuits, including the various cellular compensatory responses, is an important challenge for future research. It is very likely that different kinds of interactions between different pathways will become apparent. In any case, a systematic elucidation of the quality control network appears to be the key for the development of specific interventions that can keep mitochondria functional over longer periods of time and preserve organismal fitness and health by slowing down aging processes.

\section{Acknowledgments}

The experimental work of HDO is supported by the Deutsche Forschungsgemeinschaft, the BMBF (GerontomitoSys) and the Cluster of Excellence 'Marcomolecular Complexes'.

\section{References}

$\checkmark 1$ Fischer F, Hamann A, Osiewacz HD: Mitochondrial quality control: an integrated network of pathways. Trends Biochem Sci 2012; 37:284-292.

$\checkmark 2$ Weber TA, Reichert AS: Impaired quality control of mitochondria: aging from a new perspective. Exp Gerontol 2010;45:503-511.

$>3$ Osiewacz HD: Genes, mitochondria and aging in filamentous fungi. Ageing Res Rev 2002;28:1-18.

4 Osiewacz HD: Genetic regulation of aging. J Mol Med 1997;75:715-727.

Mitochondrial Quality Control
5 Osiewacz HD, Hermanns J: The role of mitochondrial DNA rearrangements in aging and human diseases. Aging (Milano) 1992;4:273286.

6 Wallace DC: Mitochondrial DNA mutations in disease and aging. Environ Mol Mutagen 2010;51:440-450.

7 Trifunovic A, Wredenberg A, Falkenberg M, Spelbrink JN, Rovio AT, Bruder CE, Bohlooly Y, Gidlof S, Oldfors A, Wibom R, Tornell J, Jacobs HT, Larsson NG: Premature ageing in mice expressing defective mitochondrial DNA polymerase. Nature 2004;429:417-423.
$>8$ Kujoth GC, Hiona A, Pugh TD, Someya S, Panzer K, Wohlgemuth SE, Hofer T, Seo AY, Sullivan R, Jobling WA, Morrow JD, Van Remmen H, Sedivy JM, Yamasoba T, Tanokura M, Weindruch R, Leeuwenburgh $\mathrm{C}$, Prolla TA: Mitochondrial DNA mutations, oxidative stress, and apoptosis in mammalian aging. Science 2005;309:481-484.

$\checkmark 9$ Vermulst M, Bielas JH, Kujoth GC, Ladiges WC, Rabinovitch PS, Prolla TA, Loeb LA: Mitochondrial point mutations do not limit the natural lifespan of mice. Nat Genet 2007;39: 540-543. 
10 Harman D: The biologic clock: the mitochondria? J Am Geriatr Soc 1972;20:145-147.

11 Lapointe J, Hekimi S: When a theory of aging ages badly. Cell Mol Life Sci 2010;67:1-8.

$\checkmark 12$ Luce K, Osiewacz HD: Increasing organismal healthspan by enhancing mitochondrial protein quality control. Nat Cell Biol 2009;11: 852-858.

13 Zintel S, Schwitalla D, Luce K, Hamann A, Osiewacz HD: Increasing mitochondrial superoxide dismutase abundance leads to impairments in protein quality control and ROS scavenging systems and to lifespan shortening. Exp Gerontol 2010;45:525-532.

14 Doonan R, McElwee JJ, Matthijssens F, Walker GA, Houthoofd K, Back P, Matscheski A, Vanfleteren JR, Gems D: Against the oxidative damage theory of aging: Superoxide dismutases protect against oxidative stress but have little or no effect on life span in Caenorhabditis elegans. Genes Dev 2008;22: 3236-3241.

- 15 Kowald A, Hamann A, Zintel S, Ullrich S, Klipp E, Osiewacz HD: A systems biological analysis links ROS metabolism to mitochondrial protein quality control. Mech Ageing Dev 2012;133:331-337.

16 Cantu D, Schaack J, Patel M: Oxidative inactivation of mitochondrial aconitase results in iron and $\mathrm{H}_{2} \mathrm{O}_{2}$-mediated neurotoxicity in rat primary mesencephalic cultures. PLoS One 2009; 4:e7095.

-17 Gardner PR, Raineri I, Epstein LB, White CW: Superoxide radical and iron modulate aconitase activity in mammalian cells. J Biol Chem 1995;270:13399-13405.

18 Tatsuta T, Langer T: Quality control of mitochondria: protection against neurodegeneration and ageing. EMBO J 2008;27:306-314.

19 Friguet B, Bulteau AL, Petropoulos I: Mitochondrial protein quality control: implications in ageing. Biotechnol J 2008;3:757-764.

20 Adam C, Picard M, Déquard-Chablat M, Sellem $\mathrm{CH}$, Denmat SH, Contamine V: Biologi$\mathrm{cal}$ roles of the Podospora anserina mitochondrial Lon protease and the importance of its N-domain. PLoS One 2012;7:e38138.
21 Weil A, Luce K, Dröse S, Wittig I, Brandt U, Osiewacz HD: Unmasking a temperature-dependent effect of the $P$. anserina $i$-AAA protease on aging and development. Cell Cycle 2011;10:4280-4290.

22 Fischer F, Weil A, Hamann A, Osiewacz HD: Human CLPP reverts the longevity phenotype of a fungal ClpP deletion strain. Nat Commun 2013;4:1397.

23 Taylor EB, Rutter J: Mitochondrial quality control by the ubiquitin-proteasome system. Biochem Soc Trans 2011;39:1509-1513.

24 Jeon HB, Choi ES, Yoon JH, Hwang JH, Chang JW, Lee EK, Choi HW, Park ZY, Yoo YJ: A proteomics approach to identify the ubiquitinated proteins in mouse heart. Biochem Biophys Res Commun 2007;357:731736.

25 Scheckhuber CQ, Erjavec N, Tinazli A, Hamann A, Nyström T, Osiewacz HD: Reducing mitochondrial fission results in increased life span and fitness of two fungal ageing models. Nat Cell Biol 2007;9:99-105.

26 Yang CC, Chen D, Lee SS, Walter L: The dynamin-related protein DRP-1 and the insulin signaling pathway cooperate to modulate $C$. elegans longevity. Aging Cell 2011;10:724728.

27 Ishihara N, Nomura M, Jofuku A, Kato H, Suzuki SO, Masuda K, Otera H, Nakanishi Y, Nonaka I, Goto Y, Taguchi N, Morinaga H, Maeda M, Takayanagi R, Yokota S, Mihara K: Mitochondrial fission factor Drp1 is essential for embryonic development and synapse formation in mice. Nat Cell Biol 2009;11:958966.

28 Youle RJ, van der Bliek AM: Mitochondrial fission, fusion, and stress. Science 2012;337: 1062-1065.

29 Kelly GL, Strasser A: The essential role of evasion from cell death in cancer. Adv Cancer Res 2011;111:39-96.
30 Hamann A, Brust D, Osiewacz HD: Apoptosis pathways in fungal growth, development and ageing. Trends Microbiol 2008;16:276283.

- 31 Brust D, Daum B, Breunig C, Hamann A, Kühlbrandt W, Osiewacz HD: Cyclophilin D links programmed cell death and organismal aging in Podospora anserina. Aging Cell 2010; 9:761-775.

32 Groebe K, Krause F, Kunstmann B, Unterluggauer $\mathrm{H}$, Reifschneider $\mathrm{NH}$, Scheckhuber CQ, Sastri C, Stegmann W, Wozny W, Schwall GP, Poznanovic S, Dencher NA, Jansen-Dürr P, Osiewacz HD, Schrattenholz A: Differential proteomic profiling of mitochondria from Podospora anserina, rat and human reveals distinct patterns of age-related oxidative changes. Exp Gerontol 2007;42:887-898.

33 Marzetti E, Hwang JCY, Lees HA, Wohlgemuth SE, Dupont-Versteegden EE, Carter CS, Bernabei R, Leeuwenburgh C: Mitochondrial death effectors: relevance to sarcopenia and disuse muscle atrophy. Biochim Biophys Acta 2009;1800:235-244.

34 Hiona A, Sanz A, Kujoth GC, Pamplona R, Seo AY, Hofer T, Someya S, Miyakawa T, Nakayama C, Samhan-Arias AK, Servais S, Barger JL, Portero-Otín M, Tanokura M, Prolla TA, Leeuwenburgh C: Mitochondrial DNA mutations induce mitochondrial dysfunction, apoptosis and sarcopenia in skeletal muscle of mitochondrial DNA mutator mice. PLoS One 2010;5:e11468.

-35 Hafner AV, Dai J, Gomes AP, Xiao CY, Palmeira CM, Rosenzweig A, Sinclair DA: Regulation of the mPTP by SIRT3-mediated deacetylation of CypD at lysine 166 suppresses age-related cardiac hypertrophy. Aging (Albany, N.Y.) 2010;2:914-923.

36 Hoppins S, Nunnari J: Mitochondrial dynamics and apoptosis-the ER connection. Science 2012;337:1052-1054

- $37 \mathrm{Lu} \mathrm{Y}$, Rolland SG, Conradt B: A molecular switch that governs mitochondrial fusion and fission mediated by the BCL2-like protein CED-9 of Caenorhabditis elegans. Proc Natl Acad Sci USA 2011;108:E813-E822. 